\title{
Fetal Frontal Cortex Transplanted to Injured Motor/Sensory Cortex of Adult Rats. I. NADPH-Diaphorase Neurons
}

\author{
Manuel F. Gonzalez and Frank R. Sharp \\ Departments of Neurology and Physiology, University of California, and the Veterans Administration Medical Center, San \\ Francisco, California 94121
}

\begin{abstract}
Fetal frontal cortex from 18-d-old embryonic rat brain was transplanted into cavities of juvenile host motor/sensory cortex. Two to seven months later, sections were reacted for NADPH-diaphorase (NADPH-d) enzyme histochemistry. NADPH-d-positive neurons survived in 11 of 13 grafts. All but one of the transplants had reduced numbers of these neurons, although in 3 transplants the reductions were moderate and not statistically significant. The distribution and morphology of NADPH-d neurons within most grafts was comparable to that of NADPH-d neurons in normal host cortex. At the margin of the 2 transplants with no NADPH-d neuronal perikarya, NADPH-d fibers crossed from host to transplant as far as a millimeter into the transplant, and on rare occasions, the host neurons that gave rise to these fibers were identified. This suggests that host-transplant interactions are possible. One transplant had an abnormally large number of NADPH-d-positive neurons and fibers, possibly due to selective survival of these neurons. The data reported here for NADPH-d in cortical transplants may also apply to neuropeptide $Y$ (NPY), since nearly all neocortical NPY neurons also contain NADPH-d.
\end{abstract}

Though the survival of mammalian neural tissue grafts in host brain was demonstrated earlier in this century (Dunn, 1917; Le Gros Clark, 1940), experiments showing behavioral effects of grafts have stimulated the recent interest in neural tissue transplantation research (Wallace and Das, 1982; Björklund and Stenevi, 1984). It has been demonstrated that rodent fetal cortex transplanted into injured motor/sensory cortex survives and establishes connections with host brain, and contains peptidergic neurons found in normal cortex (Ebner et al., 1984; Castro et al., 1985; Das, 1985). Whether these cortical grafts interact with host brain and function is still not known.

The demonstration that the glucose metabolic rate of surviving cortical transplants is less than that of adjacent host gray matter (Sharp and Gonzalez, 1984) suggests that transplants have abnormalities in their connections, cells, or internal organization. This may occur because some fetal cells are more

\footnotetext{
Received July 21, 1986; revised Mar. 9, 1987; accepted Apr. 14, 1987.

We wish to thank Ms. Janet Loken for technical assistance and for assisting in preparing the figures, and Dr. Stephen M. Sagar for advice on the histochemical method and comments on the manuscript. This work was supported by United Cercbral Palsy Foundation Grant R-354-85, National Science Foundation Grant BNS-8503176, and by the Research Service of the Veterans Administration.

Correspondence should be addressed to Dr. Manuel F. Gonzalez, Veterans Administration Medical Center, Department of Neurology V127, 4150 Clement Street, San Francisco, CA 94121.

Copyright (C) 1987 Society for Neuroscience $0270-6474 / 87 / 102991-11 \$ 02.00 / 0$
}

sensitive to the ischemia and trauma inherent in transplantation, or because some normal developmental changes fail to occur in transplanted fetal tissue, resulting in cell death. For example, though fetal retinae transplanted to newborn cortex survive and laminate, many retinal ganglion cells die, apparently because of their failure to make appropriate connections (McLoon and Lund, 1984).

The present work attempts to answer some of these questions by examining the presence, organization, and connections of identifiable neurons in fetal cortical transplants. This data might help explain the metabolic deficits of cortical grafts, and to assess whether they can be behaviorally functional. This report demonstrates that NADPH-d-positive neurons survive in most transplants. The following study (Sharp et al., 1987) shows that vasoactive intestinal polypeptide-, somatostatin-, and neuropeptide Y-like immunoreactive neurons survive in all transplants. Both studies show some connections between transplant and host brain.

\section{Materials and Methods}

Subjects and transplant surgery. Male Sprague-Dawley rats $(90-120 \mathrm{gm})$ were used as hosts. Fetal donor tissue was obtained from timed pregnant Sprague-Dawley rats at $18 \mathrm{~d}$ of gestation. Surgery was performed under ketamine $(80 \mathrm{mg} / \mathrm{kg})$ and xylazine $(8 \mathrm{mg} / \mathrm{kg})$ anesthesia. After incising the skin, the skull was thinned using a dentist's drill, and a bone flap cut with a scalpel blade from bregma to $3 \mathrm{~mm}$ anterior to brcgma, and from 1 to $4 \mathrm{~mm}$ lateral to the sagittal suture. In 72 subjects this flap was removed, and in 90 it was opened by gently lifting it, using the coronal suture as a hinge. The cortex under this opening was then removed by gentle suction down to the corpus callosum white matter. After the bleeding had stopped, all 72 subjects in which the bone flap was removed and 60 of the subjects with hinged flaps received grafts of donor tissue. The cavities of the remaining 30 subjects were filled with Gelfoam. The bone flaps were closed and the skin sutured. Seven days later, the 30 subjects were reanesthetized and their cavities opened. After the Gelfoam was removed, neural grafts were inserted as in other subjects.

Fetuses were removed individually from pentobarbital-anesthetized $(40 \mathrm{mg} / \mathrm{kg}$ ) pregnant rats. Solid pieces of frontal cortical tissue, about $1.5 \mathrm{~mm}^{2}$ and $1 \mathrm{~mm}$ thick, wcre dissceted using sharp scalpels. Thesc grafts included the approximate region of motor/sensory cortex dissected in hosts, but also a much greater proportion of frontal cortex. Fetal cortical tissue was transferred into the host cavity, maintaining its original orientation. A drop of $0.9 \%$ saline was placed over transplants, flaps (when present) were closed, and the wounds sutured.

NADPH-diaphorase histochemistry. Two to seven months after surgery, half of the subjects were reanesthetized with ketamine $(80 \mathrm{mg} / \mathrm{kg})$ and xylazine $(8 \mathrm{mg} / \mathrm{kg})$ and perfused via the ascending aorta with $4 \%$ paraformaldehyde in $0.1 \mathrm{M}$ PBS. Their brains were removed and 50 $\mu \mathrm{m}$-thick serial sections cut in PBS on a Vibratome. In the other half of the subjects, the fixative was followed by $200 \mathrm{ml}$ of $10 \%$ sucrose in PBS. Their brains were then removed, soaked in this solution overnight, and frozen. Coronal sections, $40 \mu \mathrm{m}$ thick, were cut at $-20^{\circ} \mathrm{C}$ in a Hacker 

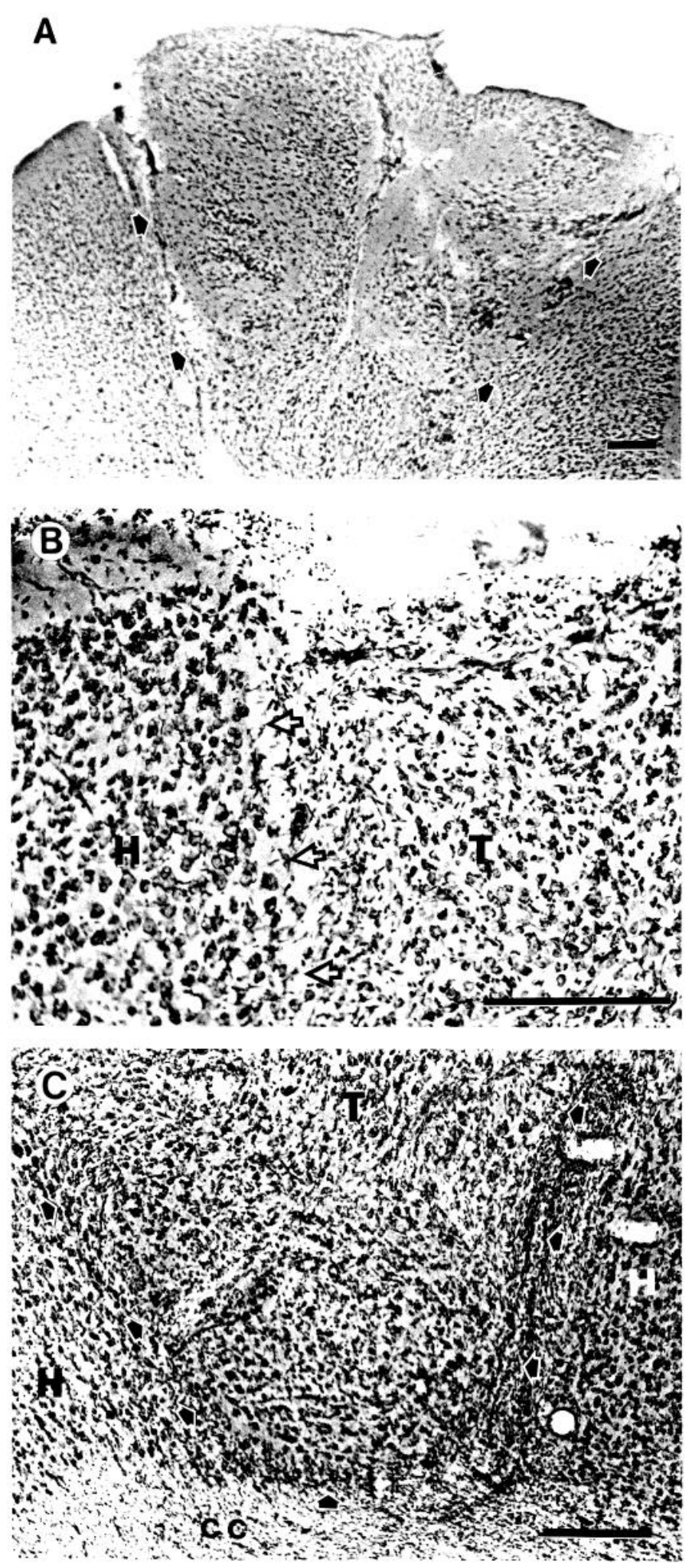

Figure 1. Cresyl violet-stained coronal sections of host $(H)$ motor cortex and transplanted $(T)$ fetal cortex. Note the absence of a molecular layer at the dorsal surface of the transplants in $A$ and $B$, but a relatively cell-free area within the transplant in $A$, which could represent abortive lamination with a molecular layer. Solid arrows in $A$ and $C$ and open arrows in $B$ point to the host-transplant interface. $c c$, Corpus callosum. Calibration bars, $250 \mu \mathrm{m}$. cryostat. These sections were picked up on a drop of PBS and air-dried on gelatinized slides at room temperature. Serial sections through the transplants or lesioned host cortices were saved. Sections cut on the Vibratome were reacted for NADPH-diaphorase (NADPH-d) in tissue culture dishes. Those cut in the cryostat were reacted on the slides in staining dishes. Both sets of sections were incubated according to the method of Sherer-Singler et al. (1983) in a $0.1 \mathrm{M}$ Tris- $\mathrm{HCl}$ ( $\mathrm{pH}$ 8) solution containing $0.2 \mathrm{~mm}$ NADP, $15 \mathrm{~mm} \mathrm{Na}$ malate, $0.2 \mathrm{~mm}$ nitro blue tetrazolium, $1 \mathrm{~mm} \mathrm{MnCl}_{2}$, and $0.1 \%$ Triton $\mathrm{X}-100$, for $1-2 \mathrm{hr}$ at $37^{\circ} \mathrm{C}$. After the reaction, free-floating sections were mounted on slides. Most of the reacted slides were then rinsed, dehydrated, and coverslipped, though some were first counterstained with neutral red.

Data analysis. Counts of the number of NADPH-d-positive neurons and their processes were made on representative sections of transplants using bright-field optics at 400 or $1000 \times$. An Apple IIe-based image analysis system was used to measure the areas of transplants and to compute the number of NADPH-d-positive neurons $/ \mathrm{mm}^{2}$. Five to fifteen sections through representative portions of each transplant were measured in each subject and averaged. The same procedure was followed in an area of equal size in normal cortex contralateral to the transplant to obtain control values. Plots of stained neuronal perikarya were made at 4 levels through the transplant of each subject on camera lucida drawings, and those showing the largest number of cells per unit area are shown in Figure 5.

\section{Results}

\section{Transplant survival}

A total of 50 surviving transplants were obtained. Twenty-one were found in rats lesioned and transplanted the same day with the skull removed, 10 in rats lesioned and transplanted the same day with skull flaps, and 19 in subjects transplanted 1 week after the lesion with skull flaps. The transplant survival rates in these 3 groups, excluding postoperative deaths, were 32,17 , and $73 \%$, respectively. The difference between these ratios was statistically significant, using an analysis of variance $(F(2,88)=13.869 ; p<$ 0.001 ), indicating a higher survival of grafts when transplantation was performed 1 week after the lesions. Sections of 22 of the 50 surviving transplants were reacted for NADPH-d activity. Of these 22 brains, 13 were reacted primarily for NADPH-d, and constitute the data of this paper. The remaining 9 NADPH-d brains were reacted primarily for peptide immunocytochemistry with selected sections reacted for NADPH-d. The results for these 9 subjects were similar to those for the 13 fully analyzed ones, but are described in the following paper (Sharp et al., 1987) because they were not analyzed quantitatively.

\section{Transplant histology and organization}

Transplants had a wide range of sizes, histological appearances, and physical linkages with the host brain. Figure 1 shows examples of surviving grafts that grew to just fill the lesion cavity. These grafts appear to have a normal number of cells of normal morphology and size. Although their cytoarchitectural organization is not normal, some transplants showed suggestions of lamination (Fig. 1A), while most did not (Fig. 1, $B, C$ ). Several types of interface between transplants and hosts were observed. Some were nearly continuous (Fig. $1, B, C$ ), some quite cellular (Fig. 1, $A, C$ ), and others had large or small gaps (Fig. 1A). In general, transplants obtained using the flap-week delay method were better fused with the host brain than those obtained using the other 2 methods.

\section{NADPH-d-positive neurons and fibers}

NADPH-d-reactive neurons were found throughout normal motor/sensory cortex (Fig. 2, $A, B$ ). Their perikarya were located in all laminae of the cortex, with the exception of the molecular 

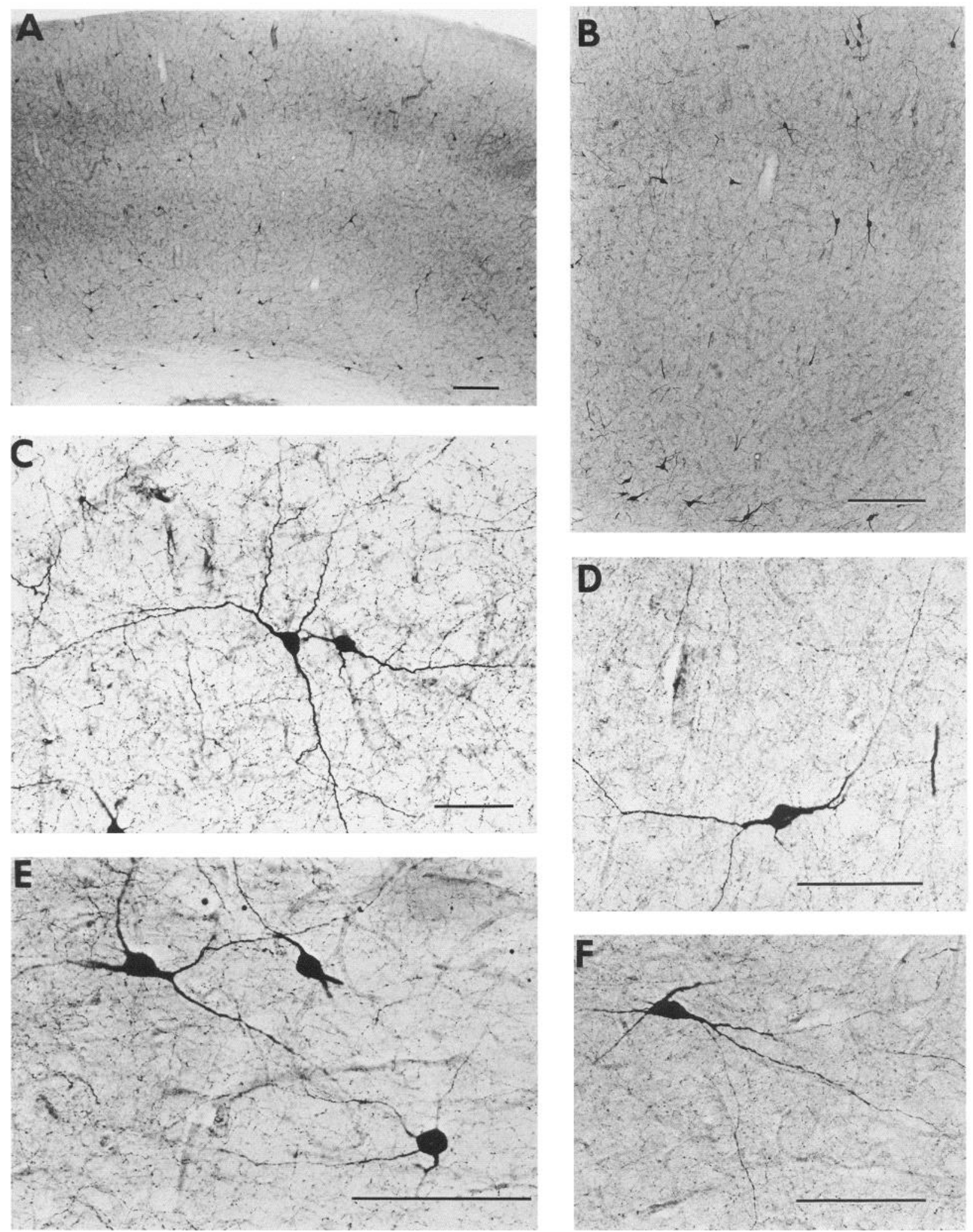

Figure 2. NADPH-d-stained neurons of normal host motor/sensory neocortex. The upper band of NADPH-d-positive neuronal fibers corresponds to cortical lamina IV, the lower band to laminae V and VI. Calibration bars, $250 \mu \mathrm{m}(A, B) ; 100 \mu \mathrm{m}(C-F)$. 

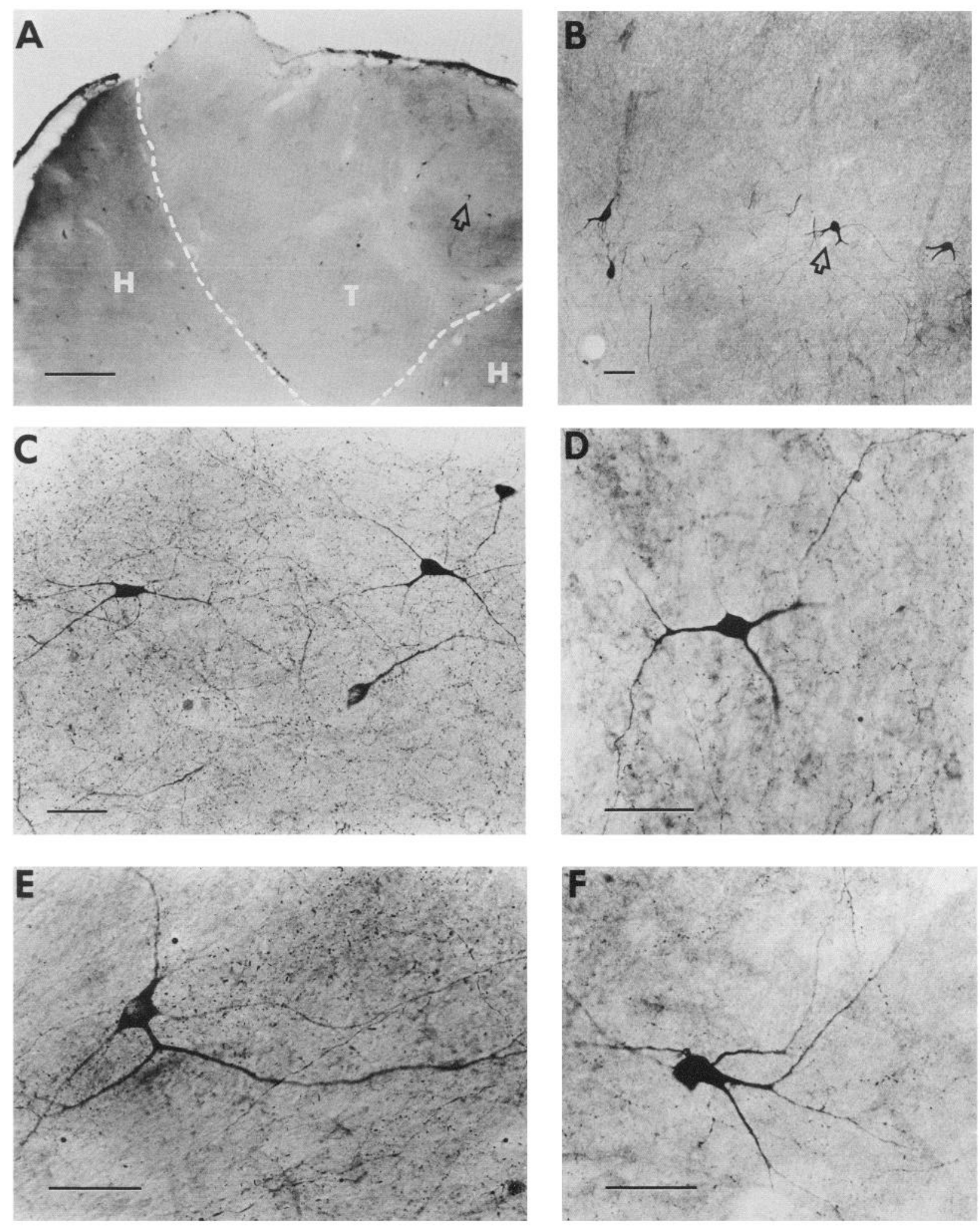

Figure 3. NADPH-d-stained neurons of fetal frontal cortex transplants $(T)$, showing examples of NADPH-d neurons within transplants with morphology comparable to that of normal neurons in Figure 2. Open arrows point to the same neurons in $A$ and $B$. $H$, Host motor/sensory cortex. Calibration bars, $500 \mu \mathrm{m}(A) ; 50 \mu \mathrm{m}(B-F)$.

layer, but appeared to be slightly more numerous in laminae II, III, and VI (Fig. 2B). Most of the perikarya were medium-sized (diameter, 10-15 $\mu \mathrm{m}$ ), with multiple branching processes at no specific orientation (Fig. $2, C, D$ ). Their processes had a beaded appearance and often could be traced for lengths of over 250 $\mu \mathrm{m}$. However, in most cases the axons could not be definitely identified (Fig. 2, C, D). The neuropil of the motor/sensory cortex showed 2 distinct bands of NADPH-d-positive fibers 
Table 1. Average number of NADPH-d-positive neurons ( \pm SEM) and average number of primary dentrites of these cells $( \pm$ SEM) in transplanted frontal cortex and in contralateral normal motor/ sensory cortex

\begin{tabular}{|c|c|c|c|c|}
\hline \multirow[b]{2}{*}{ Subject $^{a}$} & \multicolumn{2}{|c|}{ Average neurons $/ \mathrm{mm}^{2}$} & \multicolumn{2}{|c|}{ Average no. of processes per neuron } \\
\hline & Normal cortex & Transplant & Normal cortex & Transplant \\
\hline $1(7)$ & $8.74 \pm 0.57$ & $0^{* * *}$ & $3.42 \pm 0.05$ & No data \\
\hline $2(7)$ & $6.41 \pm 0.42$ & $24.69 \pm 6.40^{*}$ & $3.63 \pm 0.09$ & $3.43 \pm 0.11$ \\
\hline $3(8)$ & $3.96 \pm 0.21$ & $0.75 \pm 0.09^{* * * *}$ & $3.20 \pm 0.16$ & $3.18 \pm 0.12$ \\
\hline $8(9)$ & $3.54 \pm 0.62$ & $1.42 \pm 0.13^{*}$ & $2.72 \pm 0.17$ & $2.80 \pm 0.38$ \\
\hline $9(9)$ & $5.93 \pm 0.58$ & $3.78 \pm 0.58^{*}$ & $3.01 \pm 0.12$ & $2.75 \pm 0.09^{*}$ \\
\hline $12(11)$ & $4.91 \pm 0.43$ & $1.50 \pm 0.43^{* * *}$ & $3.57 \pm 0.18$ & $2.14 \pm 0.14^{* * *}$ \\
\hline $19(8)$ & $6.12 \pm 0.66$ & $0^{* * *}$ & $3.69 \pm 0.18$ & No data \\
\hline $53(24)$ & $6.15 \pm 0.39$ & $3.25 \pm 0.68^{* * *}$ & $2.96 \pm 0.09$ & $2.95 \pm 0.15$ \\
\hline $54(24)$ & $5.70 \pm 1.20$ & $4.16 \pm 2.17$ & $3.10 \pm 0.08$ & $3.32 \pm 0.20$ \\
\hline $55(24)$ & $4.07 \pm 0.52$ & $1.12 \pm 0.20^{* * *}$ & $2.78 \pm 0.05$ & $2.72 \pm 0.15$ \\
\hline $58(27)$ & $7.77 \pm 0.49$ & $5.93 \pm 1.68$ & $3.48 \pm 0.12$ & $2.98 \pm 0.21^{*}$ \\
\hline $59(27)$ & $6.90 \pm 1.08$ & $3.91 \pm 0.85$ & $3.40 \pm 0.10$ & $3.56 \pm 0.20$ \\
\hline $60(28)$ & $5.88+0.97$ & $1.85+0.48^{* *}$ & $3.09+0.22$ & $3.29+0.14$ \\
\hline
\end{tabular}

"Number in parentheses indicates survival time of transplants in weeks.

${ }^{*} p<0.05$ vs normal cortex; paired $t$ test. ${ }^{* *} p<0.01$ vs normal cortex; paired $t$ test. ${ }^{* * *} p<0.001$ vs normal cortex; paired $t$ test.

(Fig. 2, $A, B$ ) corresponding to cortical layer 4, and the border region between layers $\mathrm{V}$ and $\mathrm{VI}$.

Of 13 transplants, 11 contained NADPH-d-positive neurons (Table 1). Figure 3 illustrates NADPH-d-positive cells in a typical graft (Fig. 3, $A, B$ ). Enlargements of individual NADPH-d neurons in transplants (Fig. 3, $C-F$ ) showed that their multiple varicose processes were at no particular oricntations, and their somas' sizes and shapes were similar to those of mature, normal motor/sensory cortical cells. Conversely, unlike the laminar staining in normal neocortex, tiie NADPH-d-positive neuropil of transplants was frequently clustered into areas of high and/ or low staining (Fig. $3 A$ ).

Of the 13 transplants, 9 had statistically significantly fewer NADPH-d-positive neurons per unit area than the contralateral host neocortex; one showed a significant increase and 3 showed no significant differences (Table 1). The range of these values was wide, from subjects having no positive neurons (nos. 1 and 19) to one showing a 4-fold increase in number (no. 2). This contrasts with the average number of dendritic processes of these positive cells, which was remarkably constant regardless of whether the cells were in normal cortex or in frontal cortex grafts. There was, however, at least one transplant whose cells had a reduced number of processes (Table 1, no. 12). Tine number of surviving NADPH-d cells and dendrites was not related to the time elapsed after transplantation (Table 1). Correlation coefficients for survival in weeks versus the percentage of surviving cells or processes were never statistically significant, even when the aberrant values of subject no. 2 were excluded.

The spatial distribution of NADPH-d-positive neurons in representative sections from all but one of the 13 transplants is illustrated in Figures 4 and 5. Transplants that had fewer NADPH-d-positive cells, as expected, exhibited large areas devoid of these neurons (nos. 1, 3,8, 19, and 55). In other instances (nos. 9, 12, 53, 54, 59, and 60), the spatial distribution of NADPH-d neurons was very similar in transplant and host. However, in some transplants the reactive neurons were organized in abnormal clusters (no. 58). The most unusual example of this occurred in the transplant of subject no. 2 , which had 4 times the normal number of NADPH-d neurons (Table 1). They clumped together to form several dense clusters of neurons and neuropil (Fig. 6, $A-C$ ).

The density of NADPH-d-positive fibers within the transplants correlated with the number of NADPH-d-positive neurons. Those brains with near-normal NADPH-d neuronal density also appeared to have near-normal densities of NADPH-d neuropil (Fig. 3A). Large clusters of positive neurons were surrounded hy heavy clouds of deeply stained neuropil (Fig. 6), and no NADPH-d-positive fibers were seen in the center of transplants that had no positive neurons (Fig. 7D).

\section{NADPH-d fibers crossing the host-transplant interface}

Transplants that had no NADPH-d-positive neurons had many NADPH-d-positive fibers near the host-transplant interfaces. Photomicrographs of these fibers are shown in Figures 7 and 8 and in schematic form in Figure 4. The fibers extended no more than $1 \mathrm{~mm}$ into the grafts. Because there were no NADPH-dpositive perikarya in these serially sectioned transplants (Fig. 4), it is proposed that these fibers sprouted from the host cortex into the grafts. In some sections, the NADPH-d-stained fibers clearly crossed the host-transplant interface (Fig. 7, $B, C$ ), and in rare instances the neurons from which they originated could be identified in the host brain (Fig. $8 A$ ). Portions of the transplant-host interface had no crossing fibers (Fig. 7D). Fibers within the transplants appeared varicose, and, when viewed at high magnification, kncblike structures were observed protruding from the fibers (Fig. $8 B$ ). Whether these knobs const:tute dendritic spines or are synaptic boutons cannot be determined from these dată.

\section{Discussion}

Fetal rat cortex transplanted into uninjured host brain always survives using the methods of Das et al. (1979). These 100\% survival rates depend upon intact host brains, relative sterility, and little bleeding around transplants (Das, 1985). The survival of fetal tissue transplanted into lesion cavities, however, plunges to less than 30\% (Das et al., 1980). Stenevi et al. (1976) improved 

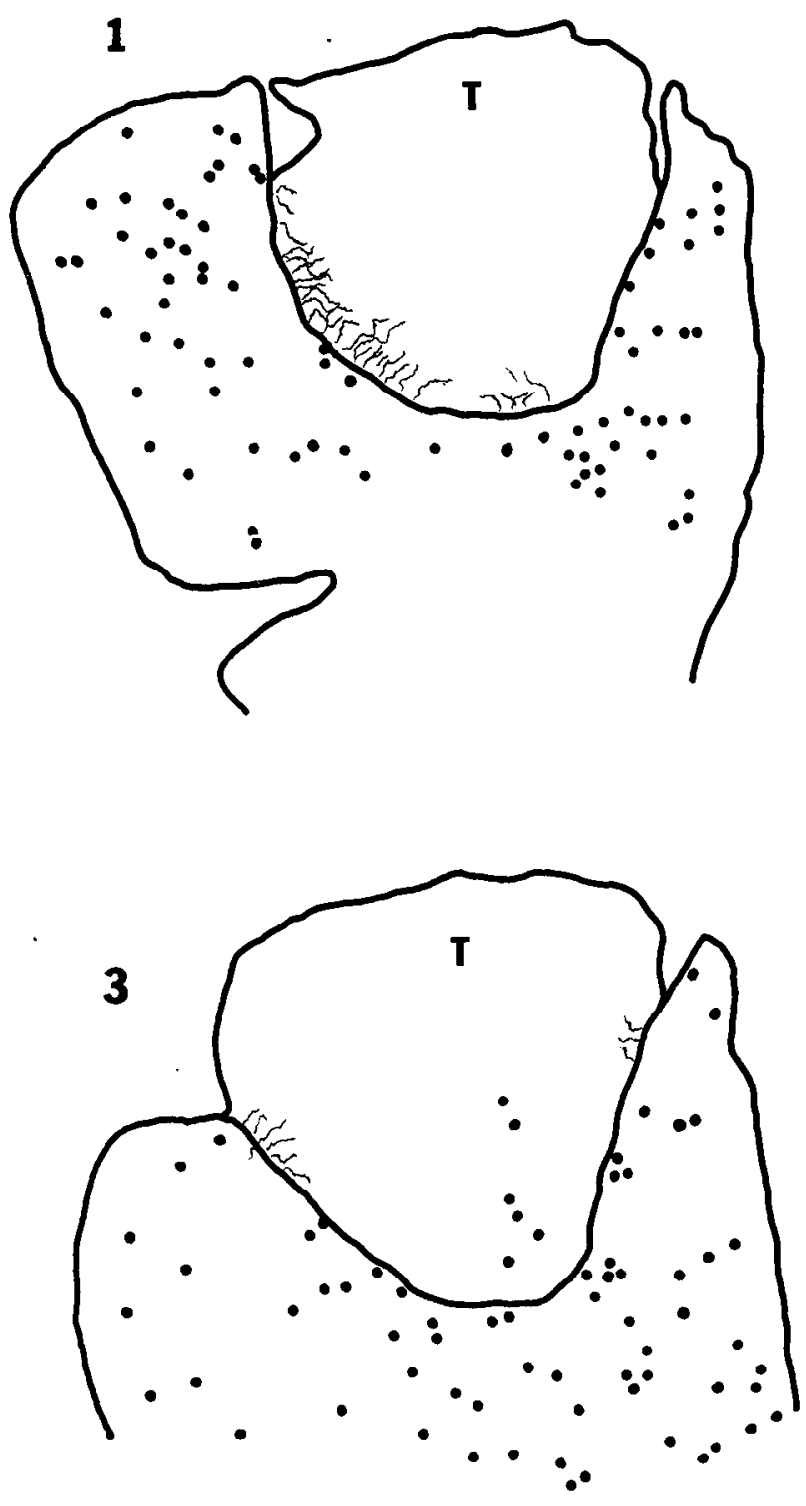

Figure 4. Camera lucida drawings of transplants $(T)$ and host cortex on which neuronal perikarya are plotted as dots and NADPH-d-positive fibers are drawn diagrammatically as wavy lines extending only within the transplant. Subject numbers are given in the upper left corner of each figure. Calibration bar, $1 \mathrm{~mm}$.

this poor transplant survival rate by placing fetal tissue into host brain cavities formed weeks to months previously. This has been confirmed many times (Björklund and Stenevi, 1979, 1984, 1985; Lewis and Cotman, 1980). Transplant survival in this study improved from 17 to $35 \%$ in tissue transplanted immediately after cavity formation to $73 \%$ in tissue transplanted after a week's delay.

Several factors could account for this improved survival, including the repair of injured blood vessels and formation of new vessels during the postlesion delay. This could have hastened the restablishment of blood flow to transplants (Stenevi et al., 1976; Rosenstein and Brightman, 1978) and helped repair a defective blood-brain barrier, which may have adversely af- fected transplants (Svendgaard et al., 1975). A second factor could be that waiting a week after host injury reduced the levels of neurotoxic substances that could have adversely affected a transplant. A third determinant of transplant survival could be trophic factors released by injured host brain. Nieto-Sampedro ct al. (1982) have shown that significant levels of ncurotrophic factors in injury cavities in adult brains appear $6 \mathrm{~d}$ postlesion and continue to increase up to $10 \mathrm{~d}$ after injury. This suggests that a delay of at least $10 \mathrm{~d}$ might optimize transplant survival. The timing of transplantation might also be critical for ultimate functional return since, while longer delays might improve transplant survival, they might also decrease the possibility that host axons sprout into the transplant. To increase transplant survival and to promote host-transplant connections, we transplanted tissue at a relatively short period following the creation of lesion cavities.

The histochemical NADPH-d enzyme reaction demonstrated positive nonpyramidal neuronal perikarya in all neocortical layers except I. They formed a small fraction $(<1 \%)$ of the total number of normal neocortical neurons (Vincent et al., 1983; Vincent, 1986). NADPH-d-positive neuronal perikarya survived in most transplants (11 of 13), and the morphology of transplanted and normal NADPH-d neurons appeared identical. The few instances where the number of dendrites on NADPH-d-positive transplanted neurons was reduced could be due to technical problems staining or counting the cell processes. This seems unlikely, since host cortex and transplant were stained and counted together. Alternatively, a subpopulation of NADPH-d neurons with multiple dendrites within the transplant could have been lost because of selective sensitivity to the ischemia of transplantation. I astly, transplantation could have caused fetal neurons to develop fewer primary dendrites during their development (McDonald et al., 1982).

Many transplants ( 9 of 13 ) had significantly fewer NADPH-d neurons $/ \mathrm{mm}^{2}$ than did contralateral host neocortex. There are several possible explanations for this. First, some NADPH-dpositive cells survived within transplants, but failed to express the enzyme. Second, some transplants had a lower proportion of neurons destined to become NADPH-d-positive. Third, in surviving transplants fewer NADPH-d neurons survived because of the trauma of transplantation. Last, the true value for the number of stained cells in host cortex is uncertain, since the average number of cells $/ \mathrm{mm}^{2}$ in host cortex varied from 3 to 9 (Table 1), reflecting the fact that somewhat different host cortical regions scrved as the control.

The NADPH-d enzyme colocalizes with neuropeptide $Y$ (NPY) and somatostatin (SS) in normal neocortical neurons (Vincent et al., 1983; Vincent, 1986). NPY cortical neurons (1) are nonpyramidal (Jones and Hendry, 1986); (2) colocalize with GABA (Jones and Hendry, 1986); (3) colocalize with NADPH-d in most neurons (Vincent et al., 1983; Sharp et al., 1987); and (4) colocalize with SS in $20-80 \%$ of neurons (Hendry et al., 1986). Though the function of NADPH-d is unknown, its colocalization with these substances may provide clues to its function. GABA is an intrinsic inhibitory neocortical transmitter found in many classes of peptidergic and nonpeptidergic neurons (Jones and Hendry, 1986). The function of NPY is uncertain, though it colocalizes with noradrenaline (NA) in superior cervical ganglion sympathetic neurons that constrict pial arteries and veins (Edvinsson et al., 1984; Ekbland et al., 1984). This, and NADPH-d fibers around blood vessels, suggests that NADPH-d/NPY neurons might affect cortical blood flow. 

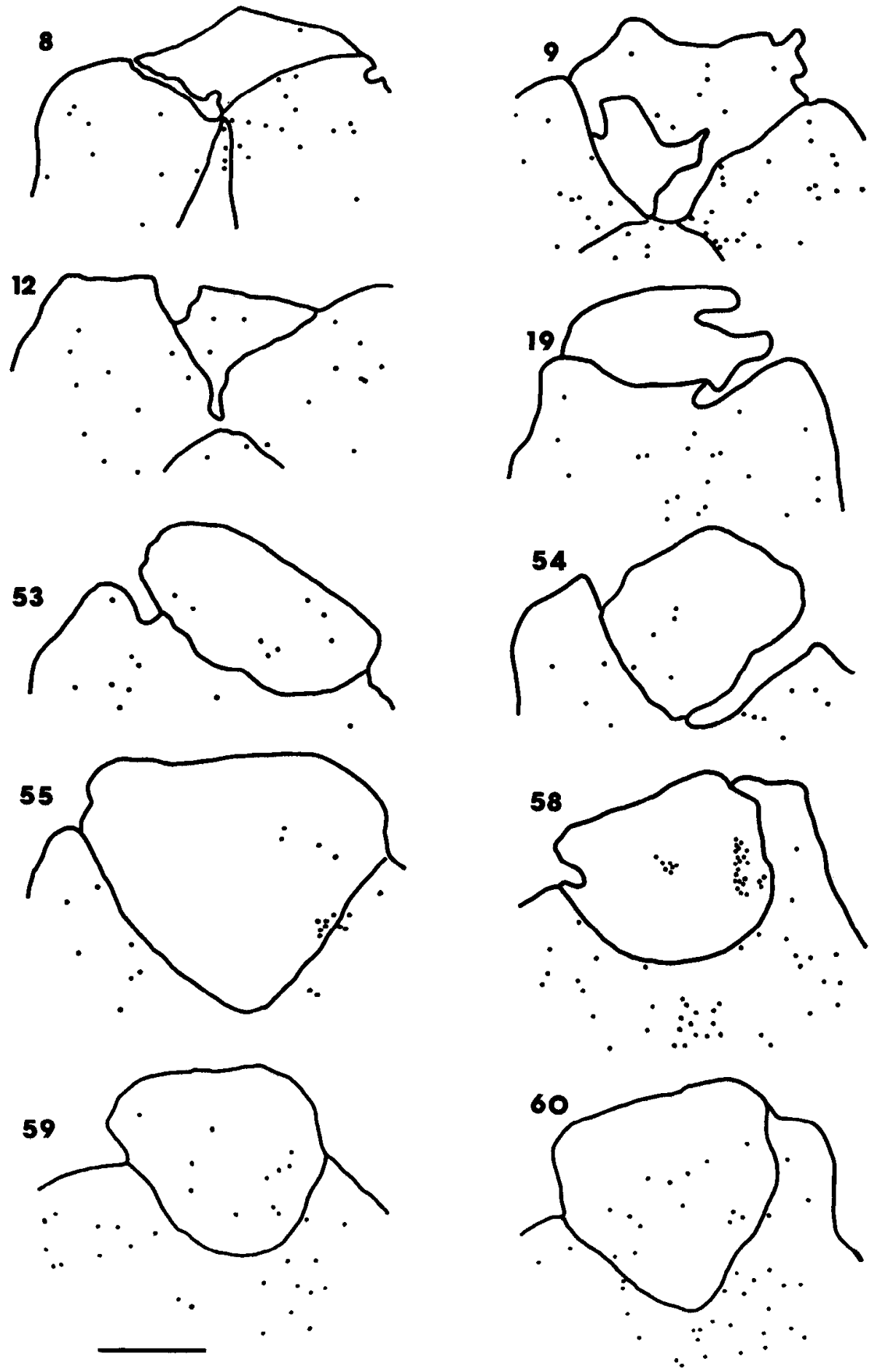

Figure 5. Camcra lucida drawings of transplants and host cortex on which NADPH-d-positive perikarya are plotted as dots. Subject numbers are given in the upper left corner of each figure. Calibration bar, $1 \mathrm{~mm}$.

The factors controlling the expression of the NADPH-d enzyme are important for interpreting the results. Some NADPH$\mathrm{d}$ - and NPY-positive fibers and cell bodies occur in the neocortex of fetal brains $18 \mathrm{~d}$ postconception (McDonald et al., 1982; Woodhams et al., 1985; Sharp et al., 1986). Since our fetal cortical transplants took place $18 \mathrm{~d}$ postconception, the trauma of transplantation could have affected the expression of NPY, NADPH-d, or both in surviving cells. The large number of surviving NADPH-d neurons in transplants suggests that most cells survived and expressed the NADPH-d enzyme. Moreover, the normal morphology of surviving NADPH-d neu- rons indicates that they continued to develop within the transplants to attain adult characteristics.

The failure to stain NADPH-d neurons in a few transplants in this study remains unexplained. NPY neurons in these grafts could have survived transplantation, but failed to express NADPH-d. This is supported by the following study (Sharp et al., 1987), which shows segments of transplants with clumps of NPY neurons that were NADPH-d-negative. Alternatively, NADPH-d neurons might have died during transplantation. A technical problem with the staining procedure is unlikely, since fibers and capillary endothelia stained within these transplants. 

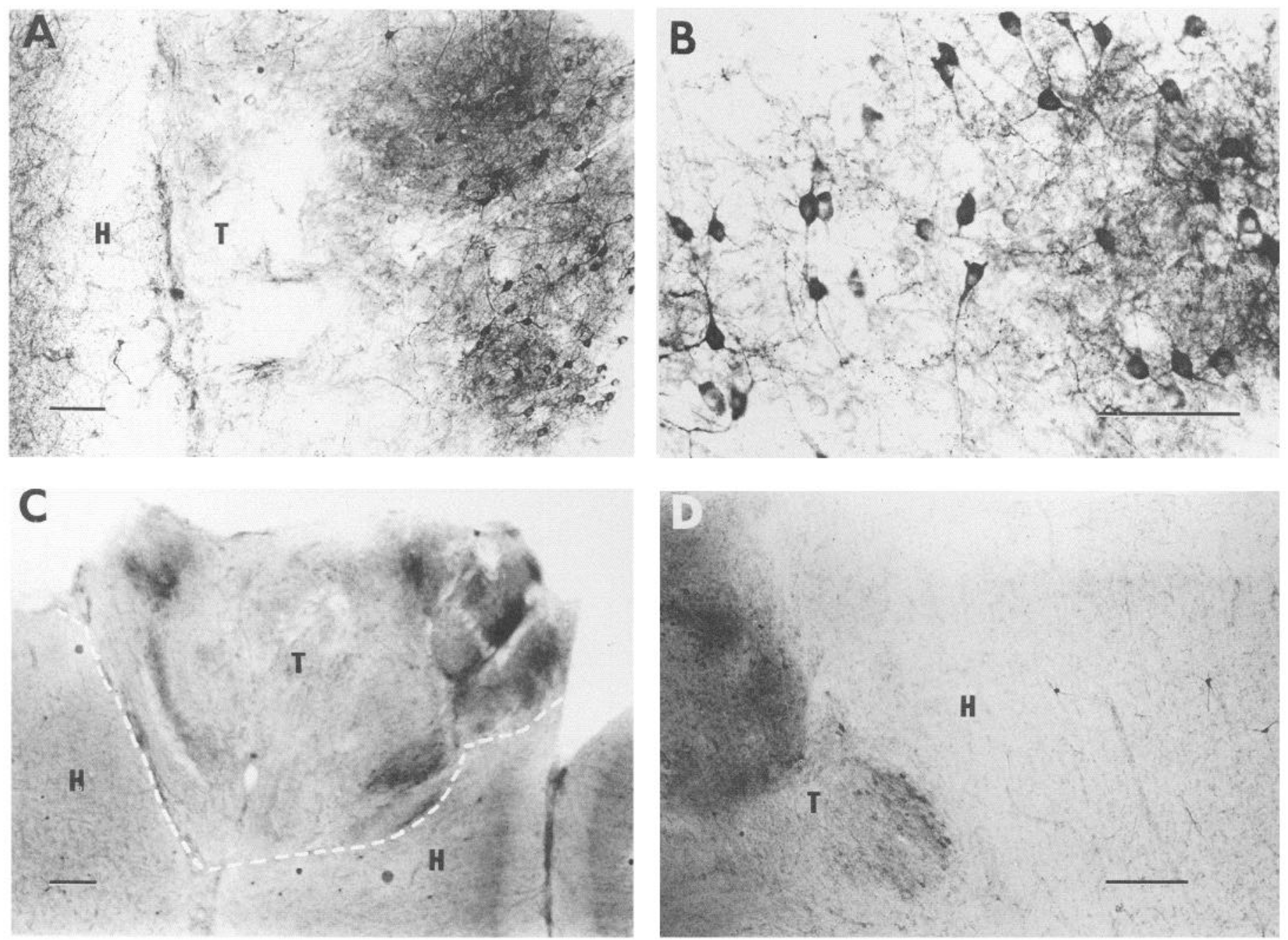

Figure 6. NADPH-d-stained sections of subject no. 2, demonstrating the abnormally dense NADPH-d clusters of positive neurons $(A, B, D)$ and fibers $(A-D)$ in the cortical transplant $(T)$ as compared with the host $(H)$ cortex. Calibration bars, $100 \mu \mathrm{m}(A, B) ; 250 \mu \mathrm{m}(C, D)$.

Last, the activity of the NADPH-d enzyme could have been altered so that staining did not occur.

The factors controlling NADPH-d enzyme expression are also important for interpreting the increased number of NADPH-d neurons in transplant 2 (Table 1; Fig. 6). This increase could be due to enzyme induction in neurons that normally do not express it. This explanation is supported by the atypical neuronal morphology of some neurons of this transplant, and by the fact that axotomy induces the expression of the enzyme in normally NADPH-d-negative neurons of the dorsal vagal motor nucleus (Gonzalez et al., 1987). NADPH-d neurons may have become malignantly transformed during the transplantation procedure, though neuronal tumors are rare and neurons have limited, preprogrammed capacities for dividing (Jaeger and Lund, 1980). Last, the NADPH-d-positive neurons may have selectively survived and grouped together in this transplant.
In the 2 transplants with no surviving NADPH-d-positive neurons, positive fibers were found at the host-transplant interface extending into the transplant for up to a millimeter. This suggests that NADPH-d neurons within the host cortex sprouted fibers into the transplant. This interpretation is supported by the facts that the serial sections of one transplant showed no NADPH-d-positive neuronal perikarya within the transplant and that positive fibers were found only at the host-transplant junction. Lastly, NADPH-d-positive neuronal perikarya within the host sent processes directly into the transplant (Fig. 8). Though this finding documents that at least some of the fibers within the transplant must be derived from the NADPH-d-positive neurons of host cortex, some fibers could have arisen from the superior cervical ganglion and traveled along blood vessels, or possibly traveled from NADPH-d-positive subcortical nuclei (Everitt et al., 1984; Vincent, 1986). 

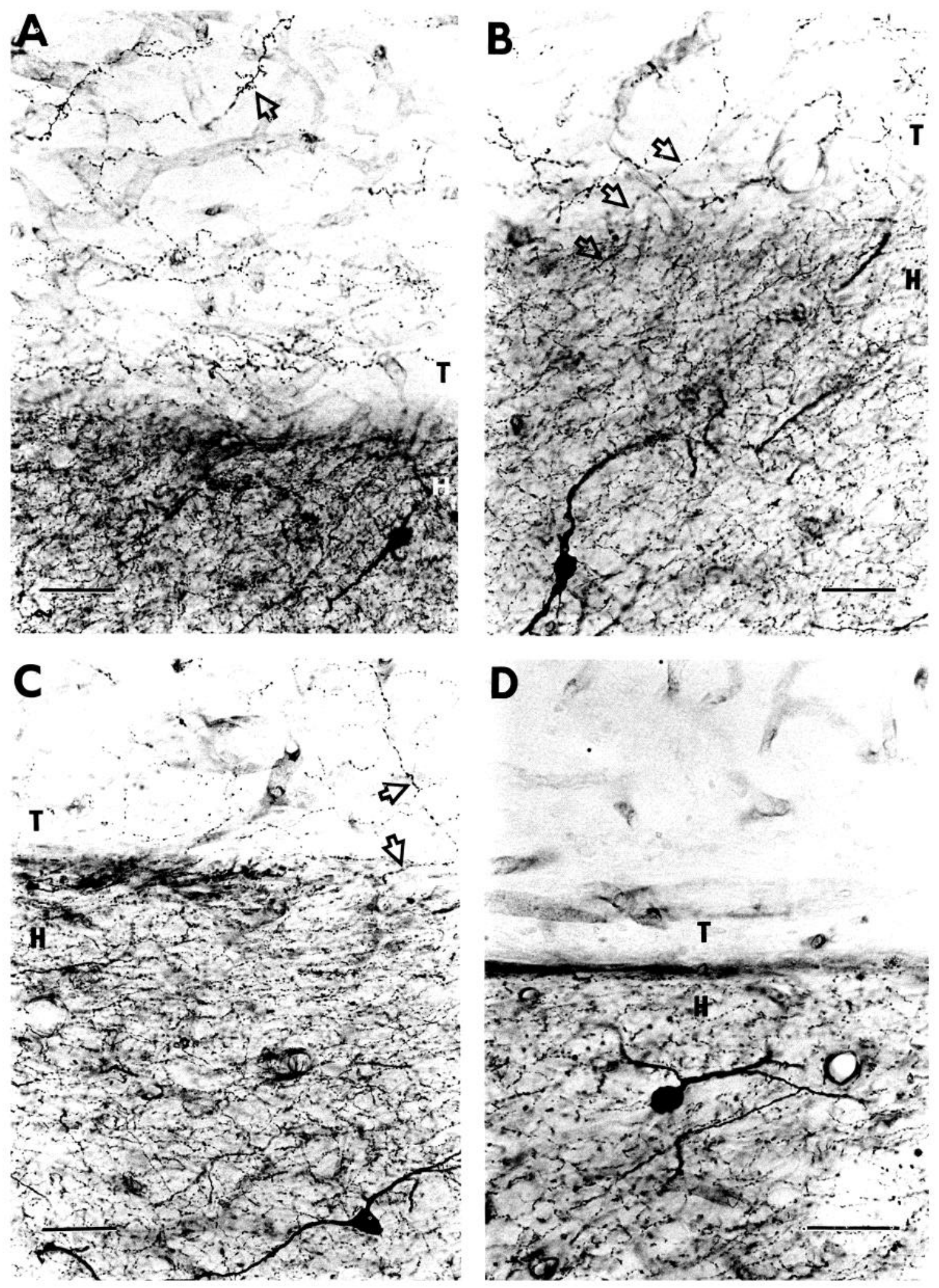

Figure 7. NADPH-d-stained sections of subject no. 1, demonstrating NADPH-d neuronal perikarya and fibers within the host $(H)$ motor/sensory neocortex $(A-D)$, the absence of NADPH-d neuronal perikarya within the transplant $(T)$, and the presence of NADPH-d-positive fibers within the transplant at some host-transplant junctions $(A-C)$ but not at others $(D)$. The open arrows in $B$ and $C$ point to fibers crossing the host-transplant junction. The open arrow in $A$ points to a fiber pictured in Figure $8 B$. Calibration bars, $50 \mu \mathrm{m}$. 

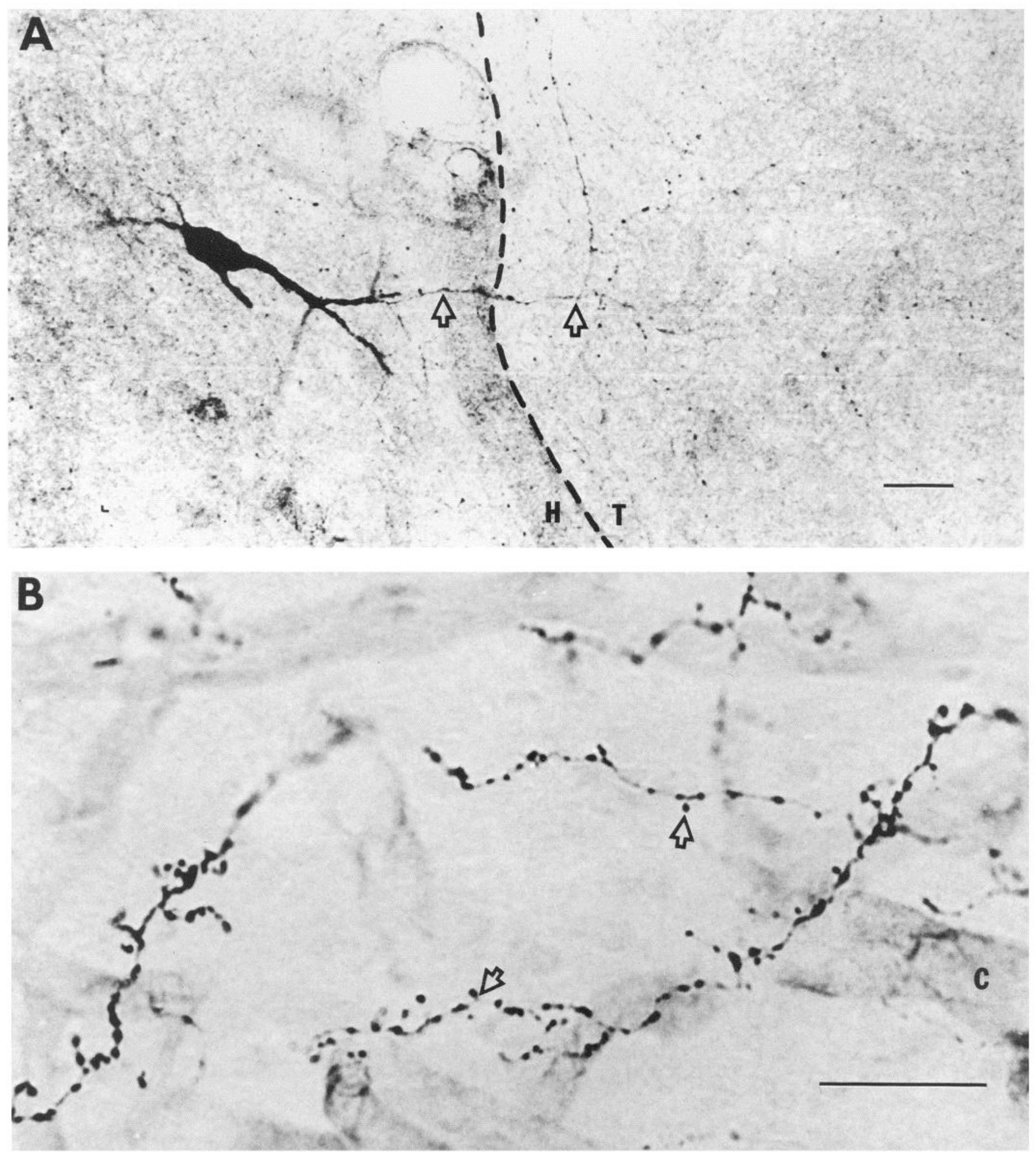

Figure 8. A, NADPH-d-stained neuronal perikaryon in the host $(H)$ cortex, which extends a fiber (open arrows) into a transplant $(T)$ ). $B$, Transplant that had no stained neuronal perikarya, but did have NADPH-d-beaded fibers with small knobs (open arrows), which could represent axonal boutons or dendritic spines. Calibration bars, $25 \mu \mathrm{m}$.

\section{References}

Björklund, A., and U. Stenevi (1979) Reconstruction of the nigrostriatal dopamine pathway in intracerebral transplants. Brain Res. 177: 555-560.
Björklund, A., and U. Stenevi (1984) Intracerebral neural implants: Neuronal replacement and reconstruction of damaged circuitries. Annu. Rev. Neurosci. 7: 279-308.

Björklund, A., and U. Stenevi, eds. (1985) Neural Grafting in the Mammalian CNS, Elsevier, Amsterdam. 
Castro, A. J., J. Zimmer, N. A. Sunde, and E. L. Bold (1985) Transplantation of fetal cortex to the brain of newborn rats: A retrograde fluorescent analysis of callosal and thalamic projections from transplant to host. Neurosci. Lett. 60: 283-288.

Das, G. D (1985) Development of neocortical transplants. In Neural Grafting in the Mammalian CNS, A. Björklund and U. Stenevi, eds., pp. 101-123, Elsevier, Amsterdam.

Das, G. D., B. H. Hallas, and K. G. Das (1979) Transplantation of neural tissues in the brains of laboratory mammals: Technical details and comments. Experientia 35: 143-153.

Das, G. D., B. H. Hallas, and K. G. Das (1980) Transplantation of brain tissue in the brain of rat. I. Growth characteristics of neocortical transplants from embryos of different ages. Am. J. Anat. 158: 135145 .

Dunn, E. H. (1917) Primary and secondary findings in a series of attempts to transplant cerebral cortex in the albino rat. J. Comp. Neurol. 27: 565-582.

Ebner, F. F., J. A. Olschowka, and D. M. Jacobowitz (1984) The development of peptide-containing neurons within neocortical transplants in adult mice. Peptides 5: 103-113.

Edvinsson, L., P. Emson, J. McCulloch, K. Tatemoto, and R. Uddman (1984) Neuropeptide Y: Immunocytochemical localization to and effect upon feline pial arteries and veins in vitro and in situ. Acta Physiol. Scand. 122: 155-163.

Ekbland, E., L. Edvinsson, C. Wahlestedt, R. Uddman, R. Håkanson, and F. Sundler (1984) Neuropeptide Y co-exists and co-operates with noradrenaline in perivascular nerve fibers. Reg. Peptides $8: 225$ 235.

Everitt, B. J., T. Hökfelt, L. Terenius, K. Tatemoto, V. Mutt, and M. Goldstein (1984) Differential co-existence of neuropeptide Y (NPY)like immunoreactivity with catecholamines in the central nervous system of the rat. Neuroscience 11: 443-462.

Gonzalez, M. F., F. R. Sharp, and S. M. Sagar (1987) Axotomy increases NADPH-diaphorase activity in vagal motor neurons of the rat. Brain Res. Bull. 18: 417-427.

Hendry, S. H. C., E. G. Jones, J. DeFelipe, D. Schmechel, C. Brandon, and P. C. Emson (1986) Neuropeptide-containing neurons of the cerebral cortex. Proc. Natl. Acad. Sci. USA 81: 6526-6530.

Jaeger, C. B., and R. D. Lund (1980) Transplantation of embryonic occipilal cortex to the brain of newborn rats. An autoradiographic study of transplant histogenesis. Exp. Brain Res. 40: 265-272.

Jones, E. G., and S. H. C. Hendry (1986) Co-localization of GABA and neuropeptides in neocortical neurons. Trends Neurosci. 9: 7176.

Le Gros Clark, W. E. (1940) Neuronal differentiation in implanted foetal cortical tissue. J. Neurol. Psychiatry 3: 263-284.

Lewis, E. R., and C. W. Cotman (1980) Mechanisms of septal lamination in the developing hippocampus revealed by outgrowth of fibers from septal implants. I. Positional and temporal factors. Brain Res. 196: 307-330.
McDonald, J. K., J. C. Parnavelas, A. N. Karamanlidis, and N. Brecha (1982) The morphology and distribution of peptide-containing neurons in the adult and developing visual cortex of the rat. IV. Avian pancreatic polypeptide. J. Neurocytol. 11: 985-995.

McLoon, S. C., and R. D. Lund (1984) Loss of ganglion cells in fetal retina transplanted to rat cortex. Dev. Brain Res. 12: 131-135.

Nieto-Sampedro, M., E. R. Lewis, C. W. Cotman, M. Manthorpe, S. D. Skaper, G. Barbin, F. M. Longo, and S. Varon (1982) Brain injury causes a time-dependent increase in neurotrophic activity at the lesion site. Science 217: 860-861.

Rosenstein, J. M., and M. W. Brightman (1978) Intact cerebral ventricle as a site for tissue transplantation. Nature 276: 83-85.

Sharp, F. R., and M. F. Gonzalez (1984) Fetal frontal cortex transplant $\left({ }^{14} \mathrm{C}\right) 2$-deoxyglucose uptake and histology: Survival in cavities of host rat brain motor cortex. Neurology 34: 1305-1311.

Sharp, F. R., M. F. Gonzalez, D. M. Ferriero, and S. M. Sagar (1986) Injured adult neocortical neurons sprout fibers into surviving fetal frontal cortex transplants: Evidence using NADPH-diaphorase staining. Neurosci. Lett. 65: 204-208.

Sharp, F. R., M. F. Gonzalez, and S. M. Sagar (1987) Fetal frontal cortex transplanted to injured motor/sensory cortex of adult rats. II VIP-, somatostatin-, and NPY-immunoreactive neurons. J. Neurosci. 7: 3002-3015.

Sherer-Singler, U., S. R. Vincent, H. Kimura, and E. G. McGeer (1983) Demonstration of a unique population of neurons with NADPHdiaphorase histochemistry. J. Neurosci. Methods 9: 229-234.

Stenevi, U., A. Björklund, and N.-A. Svendgaard (1976) Transplantation of central and peripheral monoamine neurons to the adult rat brain: Techniques and conditions for survival. Brain Res. 114: 1-20.

Svendgaard, N.-A., A. Björklund, J.-E. Hardebo, and U. Stenevi (1975) Axonal degeneration associated with a defective blood-brain barrier in cerebral implants. Nature 255: 334-337.

Vincent, S. R. (1986) NADPH-diaphorase histochemistry and neurotransmitter coexistence. In Neurohistochemistry: Modern Methods and Applications, P. Panula, H. Päivärinta, and S. Soinila, eds., pp. 375-396, Liss, New York.

Vincent, S. R., O. Johansson, T. Hökfelt, L. Skirboll, R. P. Elde, L. Terenius, J. Kimmel, and M. Goldstein (1983) NADPH-diaphorase: A selective histochemical marker for striatal neurons containing both somatostatin- and avian pancreatic polypeptide (APP)-like immunoreactivities. J. Comp. Neurol. 217: 252-263.

Wallace, R. B., and G. D. Das (1982) Behavioral effects of CNS transplants in the rat. Brain Res. 243: 133-139.

Woodhams, P. L., Y. S. Allen, J. McGovern, J. M. Allen, S. R. Bloom, R. Balazs, and J. M. Polak (1985) Immunohistochemical analysis of the early ontogeny of the neuropeptide $Y$ system in rat brain. Neuroscience 15: 173-202. 